\title{
The effect of intracameral, per-operative antibiotics on microbial contamination of anterior chamber aspirates during phacoemulsification
}

\begin{abstract}
Purpose To assess the effect of per-operative antibiotics on contamination of anterior chamber (AC) aspirates during phacoemulsification.

Methods Two hundred and twenty patients undergoing phacoemulsification of cataract were randomly allocated to receive an irrigation infusion fluid containing either balanced salt solution (BSS) alone or BSS with vancomycin $(20 \mathrm{mg} / \mathrm{l})$ and gentamicin $(8 \mathrm{mg} / \mathrm{l})$ during surgery. Conjunctival swabs were obtained from all patients immediately before pre-operative preparation. At the end of surgery $20 \mathrm{ml}$ of the AC aspirate was sent for direct and enrichment cultures. Qualitative and quantitative microbiological studies were undertaken. The chi-squared test was used to compare differences between the two groups. Results There was no significant difference between the positive culture rates of the conjunctival swabs ( 28 vs $27 ; p>0.8$ ). In the group that received BSS alone there were 22 $(20 \%)$ positive AC aspirate cultures, 18 of which were from enrichment cultures. There were 3 positive $(2.7 \%)$ cultures from the group that received antibiotics added to the BSS $(p<0.0001)$.

Conclusion The addition of gentamicin and vancomycin to the irrigation fluid during phacoemulsification results in a highly significant reduction in the microbial contamination of $\mathrm{AC}$ aspirates.
\end{abstract}

Key words Anterior chamber contamination, Endophthalmitis, Intraocular antibiotics, Phacoemulsification

Endophthalmitis is a small but serious risk of intraocular surgery. The incidence has been found to be lower for extracapsular cataract
BIJAN BEIGI, WILLIAM WESTLAKE, BERNARD CHANG, CATHERINE MARSH, JOHN JACOB, TERRY RIORDAN extraction $(0.1 \%)$ than intracapsular extraction $(0.7 \%){ }^{1}$ Recent studies in developed countries have reported an incidence as high as $0.3-0.7 \%{ }^{2,3}$ The vast number of operations performed worldwide and the serious visual morbidity arising from endophthalmitis demand that every effort be made to minimise the incidence further.

Endophthalmitis usually arises from contamination of the operating field during surgery by commensals of the ocular surface or airborne bacteria. ${ }^{4}$ These contaminate the anterior chamber (AC) either directly or by adhering to the intraocular lens. ${ }^{5}$ Endogenous infections are felt to be uncommon. ${ }^{6}$ Most, if not all, post-operative intraocular infections are caused by organisms introduced at the time of the surgery rather than subsequently acquired. ${ }^{7}$

The commonest Gram-positive causative organisms are coagulase-negative staphylococci (CNS), but a significant number of endophthalmitis cases arise from Gramnegative organisms such as Proteus sp. ${ }^{8}$ Efforts have been made to reduce per-operative intraocular microbial contamination by meticulous pre-operative ocular preparation, such as the pre-operative use of povidoneiodine ${ }^{9}$ and the administration of per-operative broad spectrum antibiotics.

We designed a prospective, randomised double-masked study to assess the effect of peroperative broad spectrum antibiotics on contamination of the AC during phacoemulsification.

\section{Materials and methods}

The conjunctival flora and microbial contamination of AC aspirates from 220 patients undergoing routine cataract extraction with phacoemulsification were studied. Patients were randomly allocated to receive one of two 
infusion fluid solutions. The theatre staff in charge of the list were responsible for randomisation by drawing colour-coded cards for each case. In group 1 (110 patients) balanced salt solution (BSS) with $1.2 \mathrm{ml} / 1$ of adrenaline $1 / 1000$ was used for infusion during surgery. In group 2 (110 patients) $20 \mathrm{mg} / 1$ vancomycin and $8 \mathrm{mg} / 1$ gentamicin were added to the BSS containing adrenaline.

Patients with ocular or general infectious disease and those who had a history of previous intraocular surgery were excluded from the study. A bacterial filter was not used with the infusion tubing. Both the surgeon and the microbiologist were masked to the allocation of each patient.

Pre-operatively guttae phenylephrine $2.5 \%$ and cyclopentolate $1 \%$ were instilled in the conjunctival sac every $15 \mathrm{~min}$ for $1 \mathrm{~h}$. Eighty per cent of operations were performed with the patient under local anaesthesia, using a peribulbar block containing a mixture of equal proportions of xylocaine $2 \%$ and bupivacaine $0.5 \%$.

Conjunctival swabs were taken from each patient prior to skin preparation. The skin was cleaned with povidone-iodine $10 \%$ and a $5 \%$ solution instilled into the conjunctival sac. An adhesive drape was used to protect the surgical field from the lashes and lid margins.

Cataract surgery was performed through a scleral tunnel using a frown incision. A $1.5 \mathrm{~mm}$ paracentesis was formed for insertion of the second instrument. After continuous circular capsulorhexis and hydrodissection, the crystalline lens was removed using the divide-andconquer phacoemulsification technique. The scleral incision was extended to $5.5 \mathrm{~mm}$ and a $5 \times 6 \mathrm{~mm}$ onepiece PMMA phaco intraocular lens (IOLAB) placed in the capsular bag. The incision site was covered by cauterising the conjunctiva at the limbus; no suture was used. Directly after each procedure $20 \mathrm{ml}$ of fluid was poured from the phaco aspiration collection cassette into a sterile specimen bottle. At the end of each operating list the AC aspirates and swabs that had been collected were sent for microbiological studies. Fifty control swabs (sterile) and 50 specimen bottles containing sterile sodium chloride were sent among the study specimens.

\section{Microbiological studies \\ Conjunctival cultures}

Conjunctival swabs were spread onto a blood agar plate (5\% Horseblood Columbia Agar base, Unipath), chocolate agar plate and a Sabouraud agar plate with gentamicin. The chocolate agar plate was incubated at $37^{\circ} \mathrm{C}$ in $5 \% \mathrm{CO}_{2}$ for $48 \mathrm{~h}$. The blood agar plate was incubated in an anaerobic cabinet for 7 days and the Sabouraud plate at $37^{\circ} \mathrm{C}$ for 7 days. An incubation temperature of $37^{\circ} \mathrm{C}$ was felt to be more suitable for detection of intraocular contaminants than the $32{ }^{\circ} \mathrm{C}$ commonly used for the study of corneal infections.
AC aspirate

AC aspirate $(0.1 \mathrm{ml})$ was inoculated by pipette onto the same types of plates as were used for the swabs, and spread with a spreader. The plates were then cultured as for conjunctival swabs (direct culture). In addition, the fluid was centrifuged and the deposit resuspended in 2 $\mathrm{ml}$ of fluid (enrichment culture). This was then inoculated into a Pedi-bact blood culture broth (Organon-Technika), supplemented with $1 \mathrm{ml}$ of Fildes extract. The bottles were incubated in a BacTAlert machine for 7 days. This is as effective as thioglycollate broth for the detection of anaerobes. Any bottles flagging positive were unloaded and subcultured both aerobically and anaerobically.

\section{Identification of isolates}

Any growth, even if scanty, was recorded and isolates were identified to genus/species level using standard methods (API kit). CNS were not identified to the species level.

\section{Statistical method}

After a pilot study, it was calculated that our sample size would have an $80 \%$ chance of producing a significant result at $p<0.05$ (EPI INFO Stat Calc). The chi-squared test was used to test for statistical significance between the two groups.

\section{Results \\ Conjunctival cultures}

Group 1 (BSS alone) had 28 (25.4\%) positive conjunctival swab cultures. Group 2 (BSS with antibiotics) had 27 (24.3\%) positive cultures. The two groups had a similar microbial flora (Tables 1, 2). There was no significant difference between the positive culture rates of the conjunctival swabs in the two groups $\left(\chi^{2}=0.02 ; p>0.8\right)$. None of the 50 control swabs was positive.

\section{AC aspirate cultures}

There were $22(20 \%)$ positive AC aspirate cultures in group 1. Six of the direct and 21 of the enriched AC aspirate cultures grew a microorganism. In group 2 there were only $3(2.7 \%)$ positive cultures, 2 of which were identified by enriched cultures. Intraocular bacterial contamination during surgery was significantly lower in this group $\left(\chi^{2}=16.29 ; p<0.0001\right)$. The types of microorganism grown in the two groups are shown in Tables 1 and 2. None of the 50 control bottles cultured positive.

\section{Discussion}

The risk of endophthalmitis is best minimised by eliminating the commensals of the ocular surface and preventing the entry of microbes into the AC during 


\begin{tabular}{|c|c|c|c|}
\hline \multirow[b]{2}{*}{ Patient no. } & \multirow[b]{2}{*}{ Conjunctival swabs } & \multicolumn{2}{|c|}{ AC culture } \\
\hline & & Direct & Enrichment \\
\hline 112 & & & Group B streptococci \\
\hline 113 & CNS & & CNS \\
\hline 114 & Acinetobacter lwoffii & & \\
\hline 115 & & CNS & \\
\hline 116 & & & Acinetobacter sp. \\
\hline 118 & CNS & & \\
\hline 123 & & & $\alpha$-haemolytic streptococci \\
\hline 128 & CNS & CNS & CNS \\
\hline 129 & & & CNS \\
\hline 130 & Morganella morganii, CNS & CNS & CNS \\
\hline 136 & CNS & $\alpha$-haemolytic streptococci, CNS & $\alpha$-haemolytic streptococci, CNS \\
\hline 138 & CNS & & \\
\hline 143 & CNS & & \\
\hline 148 & & & CNS \\
\hline 152 & & & CNS \\
\hline 156 & Oxidase + ve Gram - ve rod & & \\
\hline 157 & CNS & & \\
\hline 158 & & & CNS \\
\hline 166 & & & CNS \\
\hline 168 & CNS, oxidase + ve Gram -ve rod & & \\
\hline 169 & CNS & & \\
\hline 170 & CNS & & \\
\hline 174 & CNS & & \\
\hline 176 & Candida sp. & & CNS \\
\hline 181 & CNS & & Acinetobacter sp. \\
\hline 184 & Staphylococcus aureus & & \\
\hline 185 & CNS & & \\
\hline 187 & CNS & & \\
\hline 189 & Haemophilus influenzae & & \\
\hline 193 & $\alpha$-haemolytic streptococci & & Oxidase - ve Gram + ve rod \\
\hline 197 & Staphylococcus aureus & & \\
\hline 198 & CNS & & CNS \\
\hline 201 & & Candida sp., CNS & Candida sp., CNS \\
\hline 202 & & & CNS \\
\hline 207 & & & CNS \\
\hline 208 & Staphylococcus aureus & & \\
\hline 209 & Coliforms & & \\
\hline 210 & & & Moraxella sp. \\
\hline 212 & CNS & & \\
\hline 216 & & & CNS \\
\hline 218 & CNS & & \\
\hline 219 & $\alpha$-haemolytic streptococci & & \\
\hline
\end{tabular}

CNS, coagulase-negative staphylococci.

surgery. Per-operative intracameral antibiotics provide a second line of defence and aid the elimination of microbes that have breached other preventive measures - for example those in the $2-3 \%$ of irrigation solutions that have previously been found to be contaminated. ${ }^{7}$

Although we have noticed a poor identification rate of anaerobes, conjunctival culture of the two groups in this study showed a comparable bacterial flora preoperatively. Probably more anaerobes would have been isolated if plates had been incubated for 14 instead of 7 days. Pre-operative skin preparation with $10 \%$ povidone-iodine and instillation of a $5 \%$ solution in the conjunctival sac is effective at reducing the number of commensal organisms within the operating field ${ }^{9,10}$ and was used in both groups. Pre-operative topical antibiotics, such as norfloxacin, gentamicin and chloramphenicol, are not effective, ${ }^{11,12}$ and were not used in this study. They can produce corneal toxicity and inhibit epithelial healing. ${ }^{13}$ Sub-conjunctival antibiotics were not used; these have been shown to have no impact on post-operative inflammation and infection. ${ }^{14}$

Gentamicin and vancomycin were added to the BSS in group 2 of this study. These are broad spectrum antibiotics and provide good coverage against Gramnegative and Gram-positive organisms respectively. The estimated concentration in the AC was $8 \mu \mathrm{g} / \mathrm{ml}$ for gentamicin and $20 \mu \mathrm{g} / \mathrm{ml}$ for vancomycin. These concentrations are within the maximum inhibitory concentration (MIC) of most Gram-negative and Grampositive organisms likely to contaminate the operative field. ${ }^{15}$ Antibiotics at these concentrations are not toxic to the retina and corneal endothelial cells.

Gills et al. ${ }^{16}$ used antibiotics in the irrigation solution during cataract surgery in a large but poorly controlled 


\begin{tabular}{|c|c|c|c|}
\hline \multirow[b]{2}{*}{ Patient no. } & \multirow[b]{2}{*}{ Conjunctival swabs } & \multicolumn{2}{|c|}{ AC culture } \\
\hline & & Direct & Enrichment \\
\hline 3 & CNS & & \\
\hline 10 & CNS & & CNS \\
\hline 11 & CNS & & \\
\hline 12 & Proteus mirabilis & & \\
\hline 14 & CNS & & \\
\hline 17 & CNS & & \\
\hline 24 & CNS & & \\
\hline 27 & Moraxella sp. & & \\
\hline 29 & & & CNS \\
\hline 31 & CNS & & \\
\hline 37 & Acinetobacter lwoffii, CNS & & \\
\hline 41 & CNS & & \\
\hline 43 & CNS & & \\
\hline 46 & CNS & & \\
\hline 50 & CNS & & \\
\hline 52 & CNS & & \\
\hline 54 & CNS & & \\
\hline 59 & CNS & & \\
\hline 66 & CNS & & \\
\hline 67 & CNS & & \\
\hline 77 & Proteus sp. & & \\
\hline 79 & $\alpha$-haemolytic streptococci & & \\
\hline 86 & CNS & Pseudomonas stutzeri & \\
\hline 95 & CNS coryneforms & & \\
\hline 102 & CNS & & \\
\hline 103 & CNS & & \\
\hline 106 & CNS & & \\
\hline 109 & CNS & & \\
\hline
\end{tabular}

CNS, coagulase-negative staphylococci.

series. They reported a 1 in 20000 post-operative intraocular infection rate using gentamicin in the irrigating fluid, 1 in 9928 using vancomycin and none in 25000 when both vancomycin and gentamicin were used. Gimbel et al. ${ }^{17}$ reported no cases of endophthalmitis in a group of 4684 cataract extractions in which both vancomycin was injected into the capsular bag at the end of the procedure and gentamicin added to the irrigation solution. They showed no endothelial cell loss due to the use of antibiotics. There were no other reported complications such as retinal toxicity. These studies inspired us to select gentamicin and vancomycin for per-operative use.

Whilst these reports have encouraged the peroperative use of antibiotics during intraocular surgery, they have not addressed the potential complications adequately. Mistakes can be made during the mixing of the antibiotic solutions. It has been suggested that pharmacists are less liable to such errors than surgeons and should prepare the infusion solutions. ${ }^{18}$ A singledose commercial preparation of the antibiotics for this purpose would also be useful. Induced resistance of microorganisms because of the widespread use of antibiotics in a large population is a further concern. To quantify the benefits of per-operative antibiotic use against its potential complications a large multi-centre study is required.

Our study was not designed to assess the effect of using per-operative antibiotics on the incidence of postoperative endophthalmitis. The aim was to identify, quantify and compare the microorganisms left in situ at the end of phacoemulsification for each group. Nevertheless, the majority of cases of panophthalmitis are caused by the growth of bacterial colonies introduced into the AC or capsular bag at surgery, ${ }^{7}$ so it would be logical to expect a lower infection rate following a reduction of AC contamination. None of our patients had post-operative endophthalmitis. Case 86 in group 2 developed post-operative membranous uveitis with posterior synechiae. The per-operative AC aspirate had grown Pseudomonas stutzeri on the enrichment plate that was resistant to gentamicin. This organism is not usually a pathogen in humans.

We have demonstrated nearly a 7 -fold reduction $(95 \%$ CI: 4.47-9.92-fold reduction) in bacterial contamination of the AC as a result of the per-operative use of two antibiotics (Fig. 1). This was a highly significant reduction $(p<0.0001)$. In contrast Henry et al., ${ }^{19}$ in a much smaller study using gentamicin in the infusion fluid, failed to show a significant difference.

Endophthalmitis has been reported despite the use of antibiotics in the irrigation field. ${ }^{20}$ Indeed before the onset of this study we experienced a case of postoperative panophthalmitis from which no microorganism was isolated following the use of peroperative antibiotics. This patient was treated with broad spectrum antibiotics and subsequently achieved a visual acuity of $6 / 9$. 


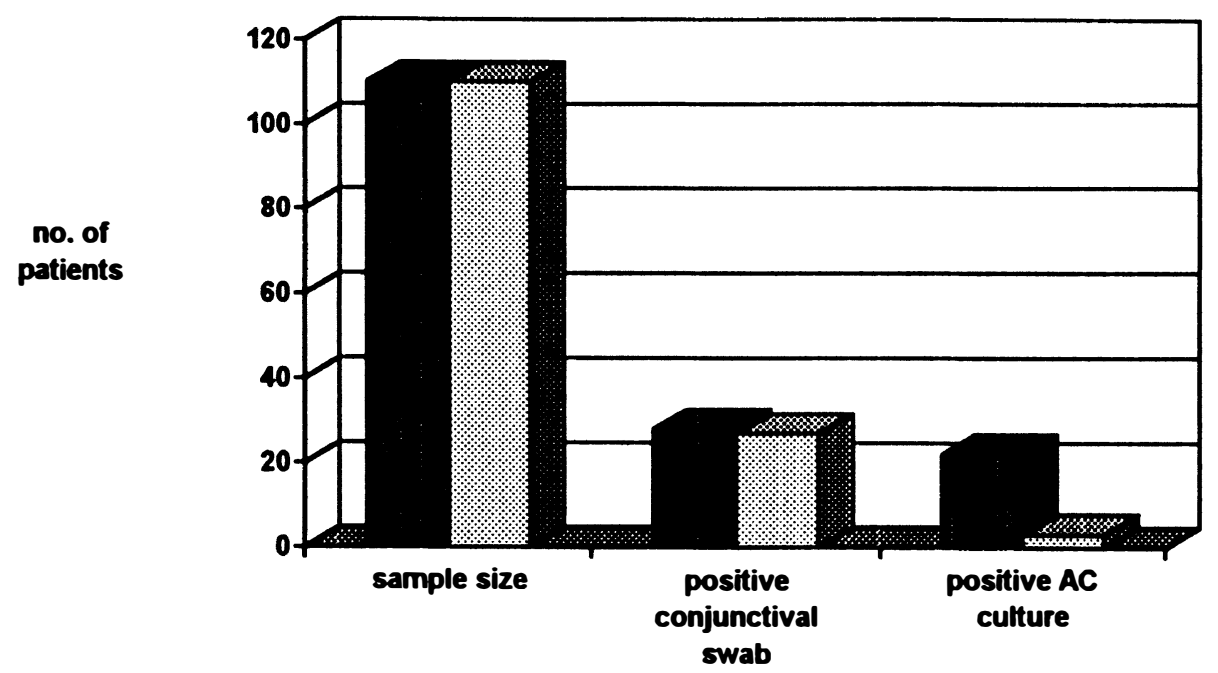

Fig. 1. Comparison of sample size, conjunctival culture and anterior chamber culture results of group 1 (no antibiotic; dark columns) and group 2 (with antibiotic; light columns).

\section{Conclusion}

The majority of cases of post-operative panophthalmitis result from the introduction of microorganisms during intraocular surgery. ${ }^{7}$ Meticulous pre-operative preparation of the ocular surface and conjunctiva by povidone-iodine, the use of adhesive sterile drapes to keep the eyelashes away from the operating field and standard protocols regarding instrument sterilisation and BSS preparation will reduce the introduction of microbes into the AC. In this study, addition of vancomycin and gentamicin to irrigation fluids reduced the AC contamination significantly (Fig. 1). The peroperative use of intracameral antibiotics is gaining popularity, although there are potential problems associated with their use. Large multi-centre studies are needed to quantify the benefits against these possible risks. On balance, the decision regarding the peroperative use of antibiotics will depend on the answer to the question: 'What price are we prepared to pay to reduce the risk of endophthalmitis to the absolute minimum?'21

The authors would like to thank all the theatre staff at the West of England Eye Unit and Mr L. Joslin in the Microbiology Department for their help during the study. We would also like to thank Mr A. Alaghebandian for his help and support.

\section{References}

1. Javitt JC, Vitale S, Canner JK, Street DA, et al. National outcomes of cataract extraction. Arch Ophthalmol 1991;109:1085-9.

2. Kattan HM, Flynn HW Jr, Pflugfelder SC, et al. Nosocomial endophthalmitis survey: current incidence of infection after intraocular surgery. Ophthalmology 1991;98:227-38.

3. Verbracken $H$. Treatment of postoperative endophthalmitis. Ophthalmologica 1995;209:165-71.

4. Sherwood DR, Rich WJ, Jacob JC, Hart RJ, Fairchild YL. Bacterial contamination of intraocular and extraocular fluids during extracapsular cataract extraction. Eye 1989;3:308-12.

5. Doyle A, Beigi B, Early A, Blake A, Eustace P, Hone R. Adherence of bacteria to intraocular lenses, a prospective study. Br J Ophthalmol 1995;79:347-9.
6. Spencer SR, Dealler SP, Hassett PDA, Todd NJ, Hawkey PM, Noble BA. Bacterial contamination of intraocular lenses: the source of the bacteria. Eye 1989;3:685-9.

7. Assalin A, Thompson P, St Antonie P, Lemire J, et al. Anterior chamber fluid contamination after uncomplicated phacoemulsification. J Cataract Refract Surg 1995;21:539-42.

8. Heaven CJ, Mann PJ, Boase DL. Endophthalmitis following extracapsular cataract surgery: a review of 32 cases. Br J Ophthalmol 1992;76:419-23.

9. Apt L, Isenberg SJ, Yoshimor R, Spierer A. Outpatient topical use of povidine-iodine in preparing the eye for surgery. Ophthalmology 89;96:289-92.

10. Klie F, Boge I, Jensen OL. The effect of polyvinylpyrrolidone iodine as a disinfectant in eye surgery. Acta Ophthalmol (Copenh) 1986;64:67-71.

11. Maeck CR, Eckardt C, Holler C. Bacteriocidal effect of preoperative use of gentamicin in comparison with PVPiodine solution. Fortschr Ophthalmol 1991;88:848-51.

12. Chitkara DK, Manners T, Chapman F, Stoddart MG, Hill D, Jenkins D. Lack of effect of the pre-operative norfloxacin on bacterial contamination of anterior aspirates after cataract surgery. Br J Ophthalmol 1994;78:772-4.

13. Caronia RM, Obstbaum SA. Drug therapy before, during and after cataract surgery. Curr Opin Ophthalmol 1993;4:61-74.

14. Sanders R, MacEwen CJ, Haining WW. A comparison of prophylactic, topical and subconjunctival treatment in cataract surgery. Eye 1992;6:105-10.

15. Kucers A, Bennett NM. Genatmicin. In: Kurcers A, Bennett NM, editors. The use of antibiotics: a comprehensive review with clinical emphasis. 4th ed. Philadelphia: JB Lippincott, 1987:629.

16. Gills JP, et al. Fiters and antibiotics in irrigating solution for cataract surgery. J Cataract Refract Surg 1991;17:385.

17. Gimbel HV, De Brof RS, De Brof BM. Prophylactic intracameral antibiotics during cataract surgery: the incidence of endophthalmitis and corneal endothelial cell loss. Eur J Implant Ref Surg 1994;6:280-5.

18. Jeglun EL, Rosenberg SB, Benson WE. Preparation of intravitreal drug doses. Ophthalmic Surg 1981;12:355-9.

19. Henry JC, Rozas D, Bryant E, Memphis W. Bacterial growth from anterior chamber fluid aspirates using different irrigating solution in phacoemulsification. Invest Ophthalmol Vis Sci 1994;34:884.

20. Alfonso EC, Flynn HW Jr. Controversies in endophthalmitis prevention: the risk of emerging resistance to vancomycin. Arch Ophthalmol 1995;113:1369-70.

21. Towler H. Aqueous contamination during small incision cataract surgery: a lesson in study design. Br J Ophthalmol 1995;79:873. 